\title{
APRENDIZAJE IDIOMA INGLÉS MEDIADO POR SUBTÍTULOS PRESENTADOS EN MEDIOS AUDIOVISUALES
}

\section{LEARNING ENGLISH LANGUAGE SUBTITLES PRESENTED IN AUDIOVISUAL MEDIA-MEDIATED}

Mayra Alejandra Bustos Rodríguez m.bustos@udla.edu.co

Universidad de la Amazonia Florencia - Colombia

Cristian Andrés Cañón Recalde c.canon@udla.edu.co

Universidad de la Amazonia Florencia - Colombia

Fotografia de portada y Diagramación Sindy Catherine Charcas Ibarra

\section{RESUMEN}

Objetivo. Desarrollar actividades de aprendizaje del idioma inglés a partir de subtítulos por herramientas audiovisuales, para medir el grado de traducción e interpretación de los estudiantes durante la actividad del aprendizaje.

Diseño. Utilizando los métodos exploratorio y proyectivo de manera conjunta con el propósito de establecer una metodología para lograr los objetivos propuestos en la investigación.

Metodología. A partir de los métodos seleccionados se definió una muestra con 55 estudiantes en tres grupos del programa de ingeniería de sistemas en la Universidad de la Amazonia en el semestre quinto, sexto y séptimo, con la colaboración de los docentes de Sistema Operativo, Ciencia y Tecnología de la Información I y Cibernética II.

Resultados. Los resultados presentaron la efectividad para el uso de subtítulos en el aprendizaje del idioma inglés, de acuerdo a los resultados, los estudiantes aprecian los medios de aprendizaje usando las nuevas tecnologías para los procesos de enseñanza.

Limitaciones. Resistencia por parte de los estudiantes al aprendizaje de una segunda lengua, requiriendo mayor atención y dedicación a partes audiovisuales de la herramienta.

Conclusión. El análisis de la efectividad para el uso de subtítulos en el aprendizaje del idioma inglés tiene viabilidad, pero se necesita tiempo, los estudiantes y docentes lo requieren para realizar las diferentes actividades, de monitoreo o seguimiento. Así mismo, se identificaron las ventajas en el uso de subtítulos como estrategia para el aprendizaje del idioma inglés, las cuales influyen por la edad, motivación, tiempo de dedicación, estrategia de enseñanza y aprendizaje, capacidad intelectual, tipos de recursos didácticos y el uso de la tecnología como apoyo en el proceso de enseñanza del aprendizaje.

Palabras clave: aprendizaje, idioma inglés, audiovisuales, subtítulos, métodos. 


\section{ABSTRACT}

Objective. To develop learning activities of the English language from subtitles by audiovisual tools, to measure the degree of translation and interpretation of students during the learning activity.

Design. Using the exploratory and projective methods joined in order to establish a methodology to achieve the objectives proposed in the research.

Methodology. Based on the selected methods, a sample was defined with 55 students in three groups of the systems engineering program at the University of Amazonia in the fifth, sixth and seventh semesters, with the collaboration of the teachers of the Operating System, Science and Technology of Information I and Cybernetics II.

Results. The results presented the effectiveness for the use of subtitles in the learning of the English language, according to the results, the students appreciate the means of learning using the new technologies for the teaching processes.

Limitations. Resistance of the students to the learning of a second language, requiring greater attention and dedication to audiovisual parts of the tool.

Conclusion. The analysis of the effectiveness for the use of subtitles in the learning of the English language is feasible but it takes time, the students and teachers require it to perform the different activities, monitoring or follow-up. Likewise, the advantages in the use of subtitles as a strategy for English language learning were identified, which influence by age, motivation, time of dedication, teaching and learning strategy, intellectual capacity, types of teaching resources and the use of technology as support in the process of teaching learning.

Keywords: learning, english language, audiovisual, subtitles, methods. 


\section{INTRODUCCIÓN}

El siguiente artículo se dará a conocer como una estrategia de aprendizaje de inglés a partir de subtítulos en medios audiovisuales basado en la necesidad de enseñar inglés de manera didáctica e innovadora. Teniendo en cuenta lo anterior, se determinó el uso de subtítulos en inglés por medio de videos con idioma español como manera para el reconocimiento del vocabulario y la compresión textual. Los métodos utilizados son el exploratorio el cual permite indagar en la literatura y contar con fuentes primarias al observar el desarrollo de los estudiantes en el aula, secundarias al indagar referentes de trabajos similares y terciarias al lograr involucrar personas expertas en el tema, el método proyectivo nos permite llevar la parte teórica a una propuesta para ser implementada en los estudiantes y ver los resultados de esta (Labajo González, 2015). Como antecedentes se pueden encontrar estrategias para desarrollar la habilidad de interactuar y aprender por medios audiovisuales, usando las herramientas tecnológicas (Abdolmanafi Rokni \& Ataee, 20l4); donde motivan a las personas a tomar la decisión de utilizar un método entretenido y hacerlo parte de su diario vivir. En el transcurso del tiempo se han desarrollado investigaciones para conocer cuál es la mejor estrategia para el aprendizaje del idioma inglés utilizando videos, imágenes u otros medios para ayudar a interactuar de forma didáctica y lúdica a los jóvenes (Acevedo Velandia \& Bustamante Bula, 2015). Iniciando el proceso de aprendizaje y reforzado las herramientas tecnológicas y el uso de los medios audiovisuales como mediadores en los procesos de enseñanza y aprendizaje (Gholami \& Mousavi, 20I4).

Mientras en algunos países la enseñanza del idioma inglés hace parte de su currículo en la educación secundaria, sin embargo, deberían enseñar el idioma inglés desde la educación básica primaria, por lo tanto, es importante aprender a temprana edad el idioma inglés, es decir, a menor edad mayor cognitiva del estudiante para aprender un idioma (Oviedo Calixto, 2016), igualmente otro tipo de estrategia es el uso de canciones, hablar con personas que dominen el idioma de la herramienta wiki (Rojas, Beatriz Palomino Hernández, \& Martha Catalina del Ángel Castillo, 2015); para el desarrollo de la destreza escrita de los estudiantes, debido a la necesidad de no solo hablarlo sino de escribirlo, esto hace a la práctica constante, mejoran la sintaxis (Barrera Calderón \& Quiroga Pineda, 2016). A partir de nuevas metodologías para el proceso de enseñanza del idioma inglés en medios audiovisuales, se usa un programa de televisión con subtítulos y tienen la oportunidad y el tiempo de traducir el diálogo visto haciendo uso del diccionario, una vez hecho esta práctica varias veces, se puede notar una mejora en los estudiantes, es decir, se familiarizan más aún con el vocabulario y no hacen tanto uso del diccionario (Williams Zambrano, Loor Fernández, Carrera Moreno, Véliz Robles, \& Congo Maldonado, 2017). Si bien es cierto a lo largo del tiempo ha surgido una evolución permanente en la tecnología, siendo esta una herramienta eficaz para el aprendizaje lúdico para estudiantes de diferentes edades, en este caso haciendo uso de recursos multimedia para los niños de preescolar y dejando atrás el método convencional de las fichas, a partir de recursos multimedia los niños pueden aprender más rápido y captar información de una manera más lúdica, porque están recibiendo información por los ojos y oídos este a su vez pueden repetir cada sesión o cambiar de contenido (Salas Machaca, 2017).

Las variables usadas con respecto al porcentaje de la actividad del manejo de videos subtitulados ayudan a desarrollar su nivel de aprendizaje hacia el inglés (Burgos Pinilla\& Maldonado Martinez, 2016). Teniendo en cuenta la cantidad de estudiantes al momento de pasar por la encuesta preliminar, la actividad y el cuestionario evaluando las capacidades de los participantes al ver el video y leer los subtítulos en inglés, como resultados preliminares se logró identificar la concordancia de los personajes con respecto en los subtítulos; sin embargo, se debe tener en cuenta variables como el tiempo del video $y$ el tipo de video, con el propósito de ofrecer una mejor experiencia al estudiante.

\section{MARCO TEÓRICO Y CONCEPTUAL}

La investigación se fundamenta en los siguientes postulados teóricos: aprendizaje idioma inglés: el aprendizaje de un nuevo idioma es de vital importancia (Chávez-Zambano, Saltos-Vivas, \& Saltos-Dueñas, 2017) porque nos permite la comunicación con diferentes personas alrededor del mundo y el inglés es el idioma internacional. Por tal motivo las instituciones educativas se están enfocando en enseñar este nuevo idioma desde temprana edad (Beltrán, 2017). Se ha vuelto un requisito en muchas empresas al momento de contratar personal (Contreras Izquierdo, 20I5).

La motivación del aprendizaje del idioma inglés desde muy temprana edad es de gran ayuda a su formación (Alvarez \& Zuluaga, 2016), por medio de las nuevas herramientas tecnológicas, estudiantes como docentes pueden interactuar dentro del aula y aprender de una manera didáctica e intuitiva (Avendaño León \& Cardenas Lozano, 2014). Es tan importante el aprendizaje del idioma inglés que el Ministerio de Educación Pública capacita a profesionales de la enseñanza para obtener un mayor número de personas dominantes de este idioma inglés (Salinas Beltrán, 20I5).

Uso de las TIC en el aprendizaje: el uso de las TIC es una herramienta fundamental en la educación, tiene como propósito el fortalecimiento de estrategias didácticas para un mejor aprendizaje, siendo los docentes quienes orienten a sus estudiantes (Gutiérrez Piñón, Herrera Sánchez, \& Pérez Nares, 2017), desde que se implementó el uso de las TIC, estudiantes como docentes han valorado positivamente el uso de estas tecnologías y han sido reflejadas en habilidades de búsqueda y selección de la información (Zuleta Hernández, 20I7).

El uso de las TIC en la educación ha surgido ventajas y desventajas, porque las nuevas tecnologías también con llevan una serie de inconvenientes en los estudiantes que abusan de ellas mismas (López, 2015). El uso de las TIC se ha convertido en parte fundamental de la enseñanza en áreas específicas como es la economía (Rivas, 2014), no obstante, también se ha desarrollado una forma interactiva de enseñar a los alumnos por medios audiovisuales $\mathrm{y}$ así acelerar su proceso 
de aprendizaje (Candel, 20I4). Por otra parte, no solo aplica en el ámbito educativo, sino que también en el ámbito artístico como lo es la música (Carranza Alcántar, Torres, \& Maciel Gómez, 2018).

\section{Medios audiovisuales para la enseñanza:} tomando como estudio de caso el (Unesco, 20l4), donde nos define: "Los Medios audiovisuales procuran abarcar el máximo de formas y formatos como las imágenes en movimiento constituyen la clásica forma de material audiovisual, sin dejar atrás las grabaciones sonoras" (p. 6).

Según estudio de caso, se pueden emplear recursos didácticos como medios audiovisuales a la enseñanza, el docente debe tener una previa preparación de la clase, preparación del ambiente, preparación del grupo de alumnos y utilización de recursos (Roncancio Cardoza, 2014). En el caso del docente tenga como apoyo los medios audiovisuales en la educación aplique la metodología activa y participativa de sus alumnos, es decir, se pueden desarrollar funciones como fomentar la participación, facilitar el aprendizaje y desarrollar la creatividad (Beltrán, Marco, 20l7).

Con respecto al marco conceptual: aprendizaje: según Thorndike (2014) expuso leyes de aprendizaje ampliamente reconocido a nivel educativo, estas leyes son conocidas por efecto "conexión Estímulo-Respuesta seguida por un refuerzo positivo o negativo", disposición "preparación de las conducciones neurológicas básicas en la formación de las conexiones Estímulo-Respuesta" (p.2). Además, enuncia el concepto de transferencia, es la adquisición de conocimiento en situaciones nuevas, donde el alumno estará expuesto a afrontar nuevos problemas siendo similares a los que se ha dominado previamente. La similitud de los contenidos facilita el aprendizaje. Así mismo, la transferencia es la solución, pues se puede descubrir elementos comunes. El alumno resolverá problemas fácilmente, cuando las situaciones son semejantes ya que toma elementos del anterior problema y lo usa como enseñanza (Barrios Vargas, Chajin Mendoza , \& Vergara Cabarcas, 2015).

Lengua Inglesa: el término "lengua internacional" tiene varios sentidos. Además de su significado original, una lengua internacional puede haber sido en origen una lengua regional, en un momento determinado, se convierte en la más hablada o en una transmisión de comunicación global. Según Halliday, el mundo actual en el que la economía ha pasado de estar basada en productos y servicios a ser una economía de la información, la lengua trasnmite dicha información, el idioma inglés, es la que se ha convertido en lengua internacional. Aparte de ser una lengua maternal en algunos países, tambien se destacan por su desarrollo tecnológico y uso de nuevos métodos de aprendizaje (Legrá Martínez, y otros, 20I5).

Audiovisuales: teniendo de referencia López de Quintana (1994), los medios audiovisuales es una información transmitida en video o audio ya sea transmitido por soportes alternativos como video, registros sonoros o diapositivas (Peralta, 2016).Es decir, si usan los medios audiovisuales para la enseñanza tendrían ventajas del uso de esos instrumentos audiovisuales como captan y es constante el interés de los educados, ayudan a los alumnos que se involucren en diversas actividades y aprendizaje autónomo (Sánchez, 2016).

Subtítulos: los títulos y los subtítulos fueron al principio usados en la instrucción de idioma extranjero en los años 1980, y unos estudios; según (Toribio Pilco , 2014) han confirmado que la combinación de títulos materiales audiovisuales es un método educacional eficaz. Según Zanon (2012), distingue tres tipos del subtitulado como sigue: subtitulado de bimodal: de diálogos ingleses; subtitulado estándar: de diálogos ingleses a subtítulos en la lengua materna de los alumnos y subtitulado invertido: de diálogos en la lengua materna de los principiantes a subtítulos ingleses (Díaz Arango \& Montes Bermúdez, 2018).

Métodos: estudia los métodos, desarrollo, explicación y justificación. Su finalidad es comprender la causa de investigación y no los resultados de la misma. se pueden tener tantas metodologías como otras formas y maneras de adquirir conocimientos científicos del saber común que se denomina "ordinario", las cuales responden de distinta manera a cada una de las preguntas y cuestionamientos planteados por la propia metodología. Se conoce varias clases de métodos aplicables a cualquier rama de la ciencia e investigación y son: Método analítico, Método sintético, Método inductivo, Método descriptivo, Método exploratorio y Método proyectivo (Labajo González, 2015).

\section{MetOdologíA}

Los procesos de la investigación científica pueden ayudar a describir, analizar y evaluar, no solo fenómenos de la naturaleza, incluso pueden enfocarse a la educación; es necesario hacer un breve recuento de los métodos utilizados en los procesos de enseñanza y aprendizaje del idioma inglés. Con énfasis en el conocimiento del método exploratorio y proyectivo; en el primero correspondió a la recolección de información mediante la aplicación de las técnicas de observaciones y entrevistas con cada sujeto en su ambiente educativo, un análisis estadístico por medio de los resultados de las encuestas, entrevistas abordadas en la investigación, respectivamente (Ramírez Rojas, 2015). El segundo método ayuda a generar una propuesta de estrategia para la enseñanza mediante los subtítulos para lo cual se recogen las experiencias obtenidas y se generó una retroalimentación ( Gavarri, 2016).

Estos nuevos enfoques educativos, los cuales contemplan diversas actividades formativas para conocer las actitudes del alumnado para el aprendizaje del idioma inglés. Está investigación se basa a partir de la detección de un problema concreto donde se le aplica un tratamiento pedagógico (Roya, R. S., Akbar, H., \& Shahla, S.20I5), ( López Barrios, 2015), se obtienen datos comprobados por encuestas y cuestionarios, estas técnicas de recolección de datos se pueden agrupar bajo tres categorías: instrumentos, estrategias y medios audiovisuales. Los procedimientos de evaluación para la investigación han obtenido el empleo de ambos métodos de investigación. A continuación, se describe en detalle los instrumentos de evaluación (Benavides Parra, 2016). 


\section{METOdOLOGÍA ESPECÍFICA}

Los objetivos de la investigación se transforman en una fase concreta de actividades a desarrollar, caracterizándose por ser novedosa y con un carácter lúdico para los alumnos, por tal motivo cada sesión se desarrolló en un tiempo mínimo de 50 minutos. Se presenta un video corto y llamativo para los estudiantes y el tiempo restante se llevó a cabo las otras actividades relacionadas a la recolección de información de los participantes. Todas las sesiones se diferencian por terminar con un resumen sobre lo realizado por los estudiantes y su percepción del ejercicio. Adicionalmente el aprendizaje del concepto se consolida con la actividad práctica.

La recolección de información consta de tres partes: la primera corresponde a la introducción, donde se realiza una encuesta preliminar para determinar el estado con respecto al manejo del vocabulario y dispositivos audiovisuales por parte de los estudiantes; la segunda parte contiene la actividad general del cortometraje junto con la estrategia utilizada (subtítulos en inglés); la tercera, es el análisis y reflexión de dicha actividad para evaluar el entendimiento de los subtítulos del cortometraje a partir de un conjunto de palabras donde unas son correctas y otras no, también se presenta una serie de palabras en inglés donde el estudiante debe darle la traducción o significado.

La recolección de datos se realizó en tres grupos de ingeniería de sistemas en la Universidad de la Amazonia, en el semestre quinto, sexto y séptimo, con la colaboración de los docentes de Sistema Operativo, Ciencia y Tecnología de la Información I y Cibernética II; el motivo del cual se tomó estos grupos fue porque a ese nivel deberían tener conocimientos hacia el inglés, y ser capaces de interactuar con la actividad en forma fluida.

Por tal motivo se obtuvo la cantidad de personas necesarias al conseguir los resultados a partir de los siguientes enunciados:

$\mathrm{N}=$ Población total de los estudiantes, se le puede aplicar la actividad, en este caso fueron 192 de 384, siendo solamente los estudiantes de 5to semestre en adelante. $Z$ = considerando una probabilidad de $4,5 \%$ (es decir $95,5 \%=2$ ) de error en la actividad realizada juntos con sus formatos de obtención de datos. $E=$ con un error muestral del $8 \%$, no resultado tan inconveniente a la hora de tomar los resultados. $p=$ tendría un valor de $0,2(2 \%)$, suponiendo algunos estudiantes tienen las características del estudio, correspondiente a aprender el idioma inglés por medio de subtítulos en inglés y audio en español. Hoy en día la mayoría de las investigaciones solo mencionado sobre el aprendizaje del inglés, pero teniendo en cuenta el audio está en idioma extranjero y los subtítulos en idioma nativo, en este caso se habla de audio en inglés y subtítulos en español. $q=$ simplemente sería la cantidad de estudiantes donde aplican el método anteriormente mencionado, en este caso sería I-p $=0,8$. Para determinar el tamaño de la muestra se aplicó la siguiente ecuación $(\mathrm{I})$ :

$$
n_{0}=\frac{z^{1} p q}{E^{2}}
$$

Al remplazar la fórmula con valores, se obtuvo la muestra del experimento desarrollado ver ecuación (2):

$$
n_{0}=\frac{2^{2} \cdot 0,2 \cdot 0,8}{0,08^{2}}=100
$$

En caso de hacer un muestreo estratificado se debe asegurar de escoger un número de elementos suficiente en cada grupo. Este tipo de muestreo no toma la población como un todo sino en varios grupos con características distintas entre ellos; de este modo se tomará el factor de corrección como la ecuación de muestreo:

$$
n=\frac{100}{1+\frac{100-1}{192}}=65,979
$$

La ecuación (3), se conoce por ser el factor de corrección, permitiendo obtener una cantidad de muestreo normalizada. Teniendo en cuenta la actividad, se redujo el número de la muestra porque se requiere tiempo en la investigación (López Flórez, 2017), se estima el intervalo de la población es del 15 a $25 \%$ aproximadamente (Vulpoiu, 2018).

\section{Desarrollo de la recolección de la información}

Para la recolección de datos y la realización de la encuesta preliminar se llevó a cabo una prueba de conocimiento al estudiante sobre algunos aspecto del inglés y la necesidad de relacionarlo con las tecnologías; en segundo lugar, se desarrolló la actividad por medio de la estrategia propuesta(los subtítulos), fue un video corto donde los estudiantes se divirtieron y usaron herramientas audiovisuales, permitiendo reconocer $y$ aprender palabras nuevas, en la tercera parte, se llevó a cabo un cuestionario, donde se evaluó a los estudiantes, sobre la atención prestada al video o las palabras nuevas y las conocidas para ellos.

Lo anterior fue aplicado a tres grupos de la carrera de ingeniería de sistemas, de semestres superiores del quinto. En horas de clases el docente permitió la realización de la actividad, en los tres grupos la cantidad fue variado, dos grupos fue de 16 y el tercer grupo fue de 23 estudiantes, en total serian 55; en el primer curso donde se desarrolló la actividad, se observó el interés en la actividad, fue dinámica porque los estudiantes mostraron sus inquietudes al momento de desarrollar el cuestionario, en el segundo y tercer curso, la dinámica fue diferente, agregando al cuestionario unas preguntas, y se le facilitó a los estudiantes realizar las actividades dichas, las sesiones duraron una hora. 


\section{Resultados y análisis}

Después de realizar las actividades prevista de acuerdo a la metodología planteada, se aplica el método y la actividad para la recolección de datos. Se generan múltiples factores estructurales influyendo la relación didáctica presente entre el docente y el estudiante. Así, son aspectos cruciales, teniendo en cuenta el número de estudiantes por grupo, el perfil del alumnado y su grado de motivación. Las condiciones como la distribución horaria a lo largo de la semana, o la disponibilidad de los espacios, dotaciones y recursos necesarios influyen en los resultados (Guillén Pinto, 2015). Todos estos aspectos afectan a la dinámica de grupo y pueden causar una percepción negativa del conjunto de la labor docente.

Hoy en día la tecnología se constituye como una instancia para apoyar situaciones, evolucionando en el mantenimiento constante entre quien enseña y quien aprende, acciones a la ayuda en pro de lograr una construcción más autónoma por parte del alumno (Benedetti \& Starc, 2014); el tiempo independiente es considerado fundamental para el trabajo de los estudiantes fuera de la clase, es por ello el método audiovisual utilizado debe ser entretenido y eficiente para el aprendizaje del estudiante. Está estrategia, se puede sistematizar y hacer parte del curso, dependiendo de las facilidades tecnológicas de la institución; para brindar a los estudiantes la oportunidad de utilizar una nueva forma de aprender. Por último, se analiza el grado de eficiencia de la estrategia propuesta, comprobando hasta donde se han cumplido los objetivos específicos.

\section{Análisis de la recolección de datos}

A continuación, se presenta el análisis de efectividad y viabilidad para el uso de subtítulos en el aprendizaje de inglés. De lo anterior, se extrae el aprendizaje realizado por el individuo, adquiriendo la relación de los nuevos conceptos y los conocimientos previos de cada estudiante. Teniendo en cuenta la estrategia presentada permite la creación de criterios sustentables de lo realizado como también el replanteamiento de conceptos adquiridos, logrando una afirmación del saber en el estudiante (Aguilar Piragua \& Molina Zubieta, 2015).

Tabla I. Cantidad de participantes por edad. Fuente: autores.

\begin{tabular}{|c|c|}
\hline Edad & Cantidad \\
\hline 18 & 3 \\
\hline 19 & 9 \\
\hline 20 & 8 \\
\hline 21 & 6 \\
\hline 22 & 5 \\
\hline 23 & 15 \\
\hline 24 & 4 \\
\hline 26 & 5 \\
\hline 50 & 1 \\
\hline
\end{tabular}

Tabla 2. Cantidad de participantes por semestre. Fuente: autores.

\begin{tabular}{|c|c|}
\hline Semestre & Cantidad \\
\hline $\mathbf{5}$ & $\mathrm{I}-5$ \\
\hline $\mathbf{6}$ & $\mathrm{I}-\mathrm{I}$ \\
\hline $\mathbf{7}$ & $\mathrm{I}-4$ \\
\hline
\end{tabular}

Se puede observar de acuerdo con la tabla I y 2, la cantidad de participantes da un total de 55 estudiantes, y el promedio de edad está entre 23 y 19 años, teniendo en cuenta los estudiantes en esta edad ya deben tener un nivel intermedio de inglés de acuerdo a la norma colombiana (Gómez Almeida, Guevara Gómez, \& Uriarte Vásquez, 2016). También, se puede observar los semestres donde se realizó la actividad conformada entre 5 to, 6to y $7 \mathrm{mo}$ semestre, deberían llevar más del $50 \%$ de las asignaturas cursadas, reafirmando lo mencionado anteriormente con respecto a la edad.

Figura I. Uso y motivación del inglés. Fuente: autores

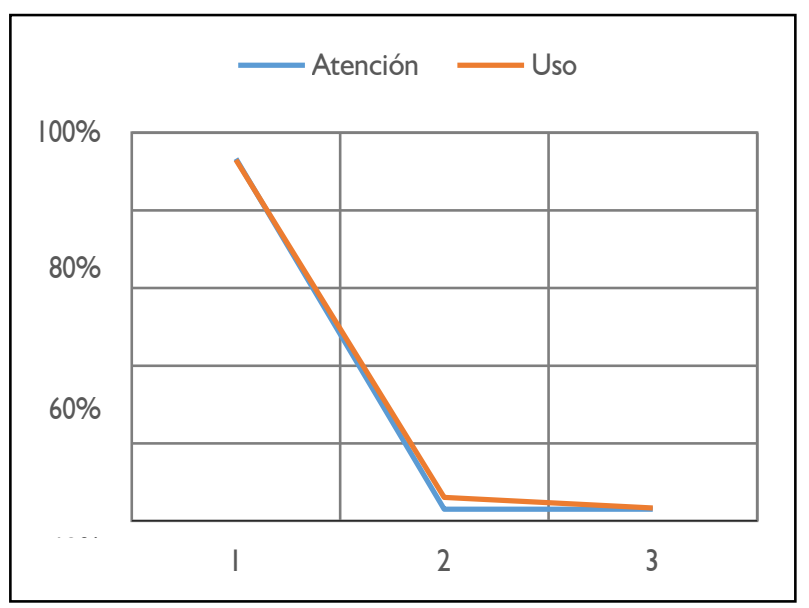

La Figura I muestra el uso y la motivación presentadas por los estudiantes con respecto al inglés; en ella se nota la similitud en ambas, mencionando los estudiantes se inclinan a decir si le dedican su tiempo, pero en realidad, los resultados obtenidos en la actividad no reflejan lo expresado por ellos (se puede ver ese resultado en la figura 2). Sin embargo, con respecto a la figura 2 se refieren a tener principalmente escaso conocimiento, es decir, si se toma el resultado de la figura anterior, se puede establecer el interés presente de los estudiantes para poder desarrollar las competencias del idioma inglés, pero se presenta la carencia de conocimiento previo para afirmar si saben del inglés lo suficiente para lo requerido en el campo profesional, como se menciona en la norma colombiana del ministerio sobre la educación superior. 
Figura 2. Nivel de conocimiento sobre el idioma inglés. Fuente: autores.

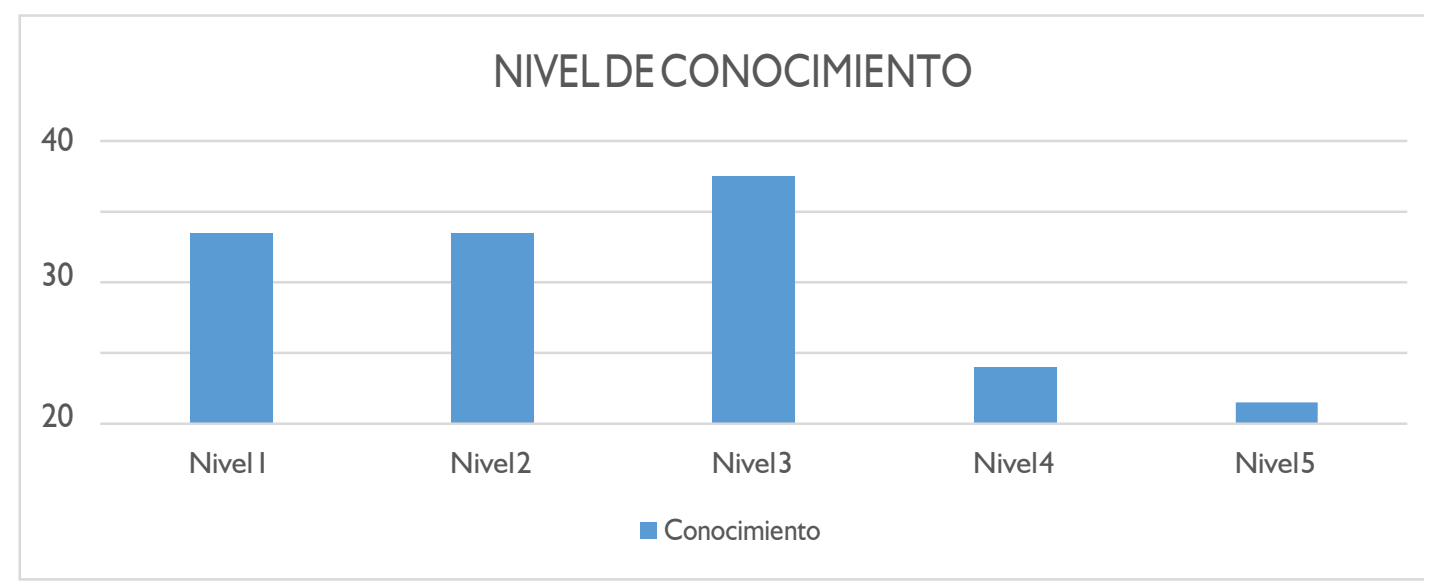

Ahora bien, refiriendo exactamente a los resultados obtenidos en la actividad con la Figura 2, se puede observar un nivel de inglés medio; los niveles I y 2 tienen un mismo porcentaje de $27 \%$ el cual refleja un nivel bajo, el nivel 3 se ubica en un $35 \%$ siendo el nivel medio, el nivel 4 se ubica en un $8 \%$ siendo el nivel sobresaliente y el nivel 5 es el nivel alto, pero obtuvo un porcentaje de $3 \%$.

Figura 3. Aprendizaje por medio de herramientas y audiovisuales. Fuente: autores.

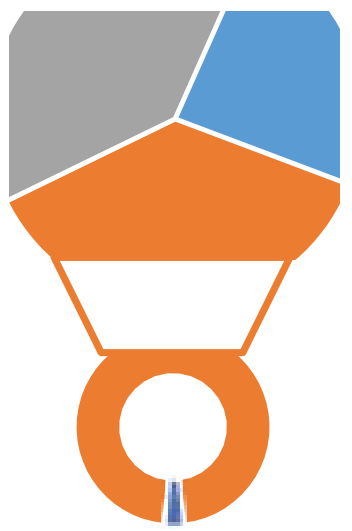

Se puede apreciar en la figura 3, como está relacionado la parte tecnológica, y medios audiovisuales en los estudiantes; entienden la importancia del aprendizaje por herramientas, ya sea por dispositivos y por medios de imágenes representadas en el video. El $62.5 \%$ consideran la importancia de las herramientas tecnológicas para el aprendizaje, están diariamente en las actividades, los resultados del aprendizaje por medios audiovisuales son de un $96 \%$, por eso se representa de forma unida indicando la relación entre ellas. Es necesario tener en cuenta dicha relación siendo primordial para el desarrollo del aprendizaje de los estudiantes, ya sea por los medios audiovisuales ayudan a mejorar o aprender dinámicamente, usando herramientas de forma rutinaria para ellos.

Tabla 3. Porcentaje de conocimiento de los estudiantes sobre los verbos del idioma inglés. Fuente: autores.

\begin{tabular}{|c|c|c|c|c|}
\hline \multicolumn{5}{|c|}{ Verbos } \\
\hline Menos $10 \mathrm{~F}$ & \multirow[t]{2}{*}{ Entre 11 y 50} & Entre 51 y 100 & Entre 101 y 150 & Más de 151 \\
\hline $25 \%$ & & $18,8 \%$ & $3,1 \%$ & $6,3 \%$ \\
\hline \multicolumn{2}{|c|}{ Medios audiovisuales } & \multicolumn{3}{|l|}{ Oraciones } \\
\hline \multicolumn{2}{|c|}{ Si } & No & \multicolumn{2}{|c|}{ más o menos } \\
\hline \multicolumn{2}{|c|}{$43,8 \%$} & $18,8 \%$ & \multicolumn{2}{|c|}{$37,5 \%$} \\
\hline \multicolumn{5}{|c|}{ Dificultades } \\
\hline \multicolumn{2}{|c|}{ Si } & \multicolumn{3}{|c|}{ No } \\
\hline \multicolumn{2}{|c|}{$40,6 \%$} & \multicolumn{3}{|c|}{$59,4 \%$} \\
\hline
\end{tabular}


Adicionalmente, se obtuvieron otros datos como lo son: Los verbos, las oraciones y las dificultades; para tener conocimiento al saber en qué nivel se encuentra los estudiantes, el desarrollo de antes y después de la actividad, así mismo ayudar a saber otros detalles para poder obtener de dificultades sobre el inglés.

Se observa en la tabla 3, en la parte superior donde muestra los porcentajes de los verbos muestra el $46,9 \%$ de los estudiantes; están en un intervalo de conocer de II a 50 verbos indicando el $25 \%$ conocen 10 verbos y la mínima parte de los estudiantes están entre 101 y 150 con un porcentaje del 3.1\%. En las oraciones, el $43,8 \%$ de los estudiantes dicen si saben hacer oraciones y la otra mitad está entre $18,8 \%$ no saben hacer oraciones y el $37,5 \%$ dicen más o menos.

Tabla 4. Importancia del uso de herramientas tecnológicas. Fuente: autores.

\begin{tabular}{|c|c|c|c|}
\hline & Correctas & $\begin{array}{c}\text { Casi } \\
\text { correctas }\end{array}$ & Incorrectas \\
\hline Pregunta I & 4 & 5 & 23 \\
\hline Pregunta 2 & 12 & 5 & 15 \\
\hline Pregunta 3 & 12 & 3 & 17 \\
\hline Pregunta 4 & 3 & 5 & 24 \\
\hline Pregunta 5 & 2 & 4 & 26 \\
\hline Pregunta 6 & 11 & 1 & 20 \\
\hline Pregunta 7 & 12 & 2 & 18 \\
\hline Pregunta 8 & 14 & 3 & 15 \\
\hline Pregunta 9 & 12 & 0 & 20 \\
\hline
\end{tabular}

En la tabla 4, se conforma de los resultados sobre las preguntas de la encuesta preliminar: La pregunta $I$ y 2 sus resultados son similares, con un porcentaje del $\mathbf{8 9 . 2 \% ~ l e s ~ l l a m a ~ l a ~ a t e n c i o ́ n ~}$ aprender inglés, teniendo en cuenta la importancia del uso del inglés en la vida cotidiana con un porcentaje del $91.9 \%$ los estudiantes están de acuerdo a la categoría del inglés.

La pregunta 3, 4 y 5, juntas se completan porque es el nivel del estudiante, aspecto importante para aprender inglés; muestran el $35.1 \%$ de los estudiantes se califican en un nivel medio y un $2.7 \%$ se consideran su nivel es excelente; hora el $62.2 \%$ de estudiantes están de acuerdo sobre las herramientas tecnológicas teniendo en cuenta el $97.3 \%$ dicen es bueno aprender inglés por medios audiovisuales.

La pregunta 6, 7 y 9, se conforman al momento del resultado porque al ver las dificultades así mismo van a hacer los resultados de la cantidad de verbos conoce y si saben conformar oraciones en inglés, teniendo en cuenta lo anterior, el $43.2 \%$ de los estudiantes tiene dificultad de aprender inglés, el $45.9 \%$ están en un intervalo entre II y 50 verbos conocidos para ellos, como resultado se tiene el $33.3 \%$ de los estudiantes maso menos saben conformar oraciones. Teniendo en cuenta lo anterior, los estudiantes prestan atención a video, pero los subtítulos los omiten; por tal razón los resultados se evidencian con un gran número de preguntas incorrectas, no obstante, algunos estudiantes se acuerdan de la idea principal, debido a momentos transcurridos durante la película, impactándolos.

Tabla 5. Atención prestada al video. Fuente: autores.

\begin{tabular}{|c|c|c|}
\hline & Correctas & Incorrectas \\
\hline Pregunta I & 17 & 15 \\
\hline Pregunta 2 & 3 & 29 \\
\hline Pregunta 3 & 9 & 23 \\
\hline Pregunta 4 & 7 & 25 \\
\hline Pregunta 5 & 2 & 30 \\
\hline Pregunta 6 & 5 & 27 \\
\hline Pregunta 7 & 3 & 29 \\
\hline Pregunta 8 & 2 & 30 \\
\hline
\end{tabular}

Teniendo en cuenta los resultados anteriores, se considera un bajo rendimiento de los estudiantes, porque estaban desubicados y entretenidos en el video; en la tabla 3, se identifica los resultados de los estudiantes, por lo tanto, no conocen muchos verbos $y$ eso influye al momento del desarrollo del cuestionario.

\section{Conclusiones}

Una vez se ha realizado el análisis e interpretación de los datos recogidos, se procede a concluir sobre el estudio del aprendizaje del inglés por medios audiovisuales, comprobando el uso de las herramientas tecnológicas como recurso eficaz en el aprendizaje del vocabulario del inglés en los jóvenes, sin embargo, la actividad se desarrolla para comprobar la utilidad de los subtítulos en inglés, obteniendo un resultado no favorable. Esto se refleja en la figura 2 con respecto al conocimiento sobre el inglés, generando dificultades para el proceso de la actividad. Entre los factores influyen el aprendizaje del inglés, en esté se encontró la edad y el semestre no intervienen en el desarrollo de estas capacidades. También el uso de recursos multimedia favorece a la medición del aprendizaje del inglés, no como el método de enseñanza tradicional usado por los docentes, pero se debe tener en cuenta al momento de llevar un seguimiento en los estudiantes indicando cómo los medios audiovisuales pueden ser de gran ayuda para su conocimiento.

Por medio de la investigación se desarrollaron los objetivos específicos, uno de ellos es: determinando el método y actividad para la identificación de las ventajas en el uso de subtítulos como estrategia para el aprendizaje del inglés, las cuales influyen edad, motivación, tiempo al idioma extranjero, estrategia de enseñanza y aprendizaje, capacidad intelectual, tipos de recursos didácticos y el uso de la tecnología como apoyo en el proceso de enseñanza del aprendizaje. Es importante tener en cuenta la aplicación del método y actividad de la recolección de datos, debido al proceso de enseñanza-aprendizaje de la comprensión auditiva, en estrecha relación con los medios audiovisuales, permite el desarrollo de habilidades referidas a lo semántico, lo gramatical. Además, constituye fuente de motivación para los estudiantes y enriquece dicho proceso de aprendizaje de la comunicación.

El análisis de la efectividad para el uso de subtítulos en el 
aprendizaje del inglés, tiene una viabilidad pero se requiere de tiempo para los estudiantes y docentes para realizar las diferentes actividades, de monitoreo o seguimiento, lo cual es comprensible por cuanto fue la primera experiencia en la utilización de este tipo de entornos para el aprendizaje del inglés; para la adaptación a esta nueva metodología para fomentar el auto- estudio y requiere de un tiempo para acceder a las actividades, puede para muchos al inicio ser inconveniente, mientras los docentes y estudiantes vayan familiarizándose con la dinámica de estudio y organicen su tiempo. Los resultados de esta investigación son útiles para docentes de diversos niveles y áreas, principalmente aquellos docentes con su área sea el inglés, a través del cual buscan mejorar la calidad de su enseñanza y del aprendizaje de sus estudiantes por medio de recursos audiovisuales.

Los subtítulos presentados en medios audiovisuales permiten inferir acerca del contenido temático, contextualizar el tema, facilitar la comprensión lectora e incluso realizar procesos de intertextualidad implícitos en el desarrollo del texto. Así mismo, presentan un avance de la temática abordada. 


\section{Referencias bibliográficas}

BIBLIOGRAPHY Alvarez, J., \& Zuluaga, T. (2016). La Motivación En El Aprendizaje Del Inglés Como Segunda Lengua.

Acevedo Velandia, J., \& Bustamante Bula, R. (2015). Uso de las WebQuests para la Enseñanza del Inglés en Estudiantes de Secundaria. Universidad de San Buenaventura.

Beltrán, Marco. (2017). El aprendizaje del idioma inglés como lengua extranjera. Universidad Técnica de Cotopaxi, 8.

Carranza Alcántar, M., Torres, C., \& Maciel Gómez, M. (2018). Percepción de los estudiantes respecto del uso de las TIC y el aprendizaje del idioma inglés. Revista Apertura, 63.

Roncancio Cardoza, D. (20I4). Desarrollo de competencias comunicativas en inglés mediadas por las TIC de un grupo de estudiantes entre los 12 y 15 años del colevio Litecom del Municipio de Jamundí, Valle del Cauca. Pontificia Universidad Javeriana Cali., 91.

Zuleta Hernández, Y. V. (20I7). Las TIC como mediación para la enseñanza y aprendizaje del idioma inglés. Universidad Pontificia Bolivariana, 77.

Aguilar Piragua, A. L., \& Molina Zubieta, L. (20I5). Rutas del aprendizaje autónomo del inglés como L2 en la era digital. Universidad Distrital Francisco José de Caldas., I 31 .

Abdolmanafi Rokni, S. J., \& Ataee, A. J. (20I4). Peliculas en aulas EFL: con o $\sin \mathrm{s} \quad$ a $\quad$ subtítulos. La revista del amanecer, 3.

Avendaño León, L. J., \& Cardenas Lozano, C. (20/4). Implementación de las Tecnologías de la Información y Comunicación (TIC `S) en la enseñanza del inglés como lengua extranjera para optimizar y favorecer el desarrollo lexical del estudiante del Ciclo Cuarto del Colegio Miguel Antonio Caro jornada Nocturna . Universidad Libre de Colombia., 13.

Barrera Calderón, V. J., \& Quiroga Pineda, S. (2016). Uso de material audiovisual: Desarrollo de la comprensión escucha en un grupo de estudiantes del ciclo II -Jornada de la noche de una Institución Educativa Distrital. Universidad Libre, 82.

Barrios Vargas , C., Chajin Mendoza , O., \& Vergara Cabarcas, L. (2015). Reetos de la enseñanza - Aprendizaje del idioma inglés en Cartagena, Colombia. Las visiones de los practicantes. Unicolombo.

Benedetti, L., \& Starc, M. (2014). La utilización de las Tic en la clase de español como lengua extranjera. Universidad del Salvador. Burgos Pinilla-, W., \& Maldonado Martinez, H. N. (2016). Medios Audiovisuales: Una estrategia de aprendizaje de inglés para los niños de preescolar del colegio la candelaria. Univesidad Libre, 89.

Candel, E. C. (20I4). Los medios audiovisuales y las TIC como herramientas para la docencia en educación secundaria. Análisis aplicado de una práctica docente. UCLM.

Chávez-Zambano, M., Saltos-Vivas, M., \& Saltos-Dueñas, C. (2017). La importancia del aprendizaje y conocimiento del idioma inglés en la enseñanza. dominio de la ciencia.

Contreras Izquierdo, N. (20I5). Ini Inv, e3: a4 (2008) i La enseñanza-aprendizaje de lenguas extranjeras y las TICs: el caso del Español como Lengua Extranjera (ELE) . Universidad de Jaén.

Díaz Arango, V., \& Montes Bermúdez, D. (2018). Los recursos y el estudiante: los Medios Audiovisuales en el aprendizaje del inglés. Universidad Pontificia Bolivariana, 133.

Gavarri, S. (20I6). El aprendizaje de lenguas extranjeras mediado por las TIC: aprender Inglés.

Gómez Almeida, L., Guevara Gómez, P., \& Uriarte Vásquez, A. (2016). Programa educativo youtube en el mejoramiento de la expresión oral en inglés en los estudiantes del primer grado de secundaria de la Institución Educativa № 60188 Simón Bolívar, Iquitos - 2016. Facultad de Ciencias de la Educación y Humanidades.

Gutiérrez Piñón, N., Herrera Sánchez, S., \& Pérez Nares, Y. (2017). Las TIC en la enseñanza del inglés en educación básica. Revista Electrónica sobre Tecnología, Educación y Sociedad , 13.

Gholami, J., \& Mousavi, F. (2014). Effects of Watching Flash Stories with or without 
Subtitle and Reading Subtitles on Incidental Vocabulary Acquisition. Procedia Social and Behavioral Sciences, 98, 8.

Gorjian, B. (2014). The effect of movie subtitling on incidental vocabulary learning among EFL learners. Department of EFL, I0I31026.

Guillén Pinto, A. (2015). El e-learning como herramienta de apoyo educativo en el trabajo colaborativo de estudiantes universitarios en modalidad presencial. Uniminuto.

Benavides Parra, M. Z. (20/6). Propuesta Educativa para el aprendizaje del inglés mediante el uso del serious games "Monsters inc: Scard Island" en estudiantes de grado Quinto de primaria de la fundación educativa Don Bosco. Universidad Militar Nueva Granada, 157.

Labajo González, E. (2015). Uiversidad Complutense Madrid. Obtenido de www.ucm.es

Legrá Martínez, N., Vaca Sánchez, A., Sagó Pérez, A., Garzón Gums, E., López Molina, E., Crespo Rivero, M., \& Lewis Goulbourne, G. (20I5). La lengua inglesa y el mundo actual. Facultad de Ciencias Medicas Guantanamo.

López, C. (2015). Uso de las TIC para el aprendizaje del inglés en la Universidad Autónoma de Aguascalientes. Universidad Autónoma de Aguascalientes.

López Flórez, F. G. (2017). Uso de tres medios didáctivos audiovisuales para motivar el aprendizaje del inglés. Universidad De La Salle, 39.

López Barrios, M. (2015). Aprendizaje Significativos con TIC en la Educación Superior. Facultad de Lenguas, Universidad Nacional de Córdoba.

Oviedo Calixto, L. (2016). Uso de los recursos audiovisuales y su influencia en el aprendizaje del idioma inglés en los estudiantes del Instituto de Educación Superior Tecnológico Aeronáutico Surco-Lima. Universidad César Vallejo, I56.

Peralta, W. (2016). Estrategias de enseñanza aprendizaje del inglés como lengua extranjera. Revista de la Educación.

Ramírez Rojas, V. (20I5). El inglés como lengua extranjera desde un enfoque comunicativo y aplicando estrategias de aprendizaje. Uniminuto.

Rivas, R. G. (20I4). Estrategias para el uso de las TIC en los procesos de aprendizaje. Universidad COMPLUTENSE Madrid.

Roya, R. S., Akbar, H., \& Shahla, S. (20I5). Effects of pedagogical movie english subtitles on EFL learners vocabulary improvement. English department, najafab branch,islamic azad university, 47-56.

BIBLIOGRAPHY Rojas, V. L., Beatriz Palomino Hernández, \& Martha Catalina del Ángel Castillo. (2015). El inglés como lengua extranjera desde un enfoque comunicativo y aplicando estrategias de aprendizaje. Uniminuti.

Salas Machaca, M. (2017). Los materiales audiovisuales para mejorar la habilidad de conversación "Speaking" en el aprendizaje del inglés en los estudiantes del nivel básico del Centro de Idiomas de la Universidad Nacional de San Agustín del Distrito de Arequipa 2017, 102.

Salinas Beltrán, Y. A. (20I5). La enseñanza “audiovisual” del inglés. Universidad Pedagógica Nacional., 108.

Sánchez, A. R. (2016). Obstáculos en el aprendizaje del inglés como lengua extranjera en dos grupo de población Bogotá. Universidad Distrital Francisco José de Caldas, 95.

Toribio Pilco , M. (2014). Los recursos audiovisuales y el aprendizaje del idioma inglés en los estudiantes del centro de idiomas San Marcos, S.J.L. Universidad César Vallejo, II.

Unesco. (2014). Estrategía de Educación de la Unesco.

Vulpoiu, E. (2018). La traducción audiovisual y el perfil del traductor audiovisual en Rumanía.

Williams Zambrano, M. S., Loor Fernández, M. N., Carrera Moreno, G. W., Véliz Robles, F. M., \& Congo Maldonado, R. (2017). Recurso didactivos audiovisuales y su impacto en el aprendizaje del idioma inglés. Didáctica y Educación, 18. 